Philosophie ANTIQUE

\section{Philosophie antique}

Problèmes, Renaissances, Usages

$10 \mid 2010$

Philosophie et mathématiques

\title{
Lisa ATWOOD WILKINSON, Parmenides and To Eon. Reconsidering Muthos and Logos
}

Continuum International Publishing Group, London-New York 2009, 176

p., ISBN 978-1-84706-245-1, € 108,60

Dario Zucchello

\section{OpenEdition}

12 Journals

Edizione digitale

URL: https://journals.openedition.org/philosant/2363

DOI: $10.4000 /$ philosant.2363

ISSN: 2648-2789

Editore

Éditions Vrin

\section{Edizione cartacea}

Data di pubblicazione: 30 octobre 2010

Paginazione: $274-278$

ISBN: 978-2-7574-0179-8

ISSN: 1634-4561

Notizia bibliografica digitale

Dario Zucchello, «Lisa ATwood WILkInson, Parmenides and To Eon. Reconsidering Muthos and Logos »,

Philosophie antique [En ligne], 10 | 2010, mis en ligne le 11 juillet 2019, consulté le 10 décembre 2022.

URL : http://journals.openedition.org/philosant/2363; DOI : https://doi.org/10.4000/philosant.2363

\section{c) (i) (9)}

Creative Commons - Attribution - Pas d'Utilisation Commerciale - Pas de Modification 4.0 International - CC BY-NC-ND 4.0

https://creativecommons.org/licenses/by-nc-nd/4.0/ 
repose tout l'argument d'Aristote. Il en vient ainsi à des énoncés comme celui-ci (p. 105) : "But only hypothetically, he agreed that infinity might exist if it were somehow possible (e.g., if his God, the UM, were powerful enough to actualize infinity), since he said that if bisection could have been carried out indefinitely, "nothing impossible will have resulted if it [a body] has actually been divided - not even if it has been divided into innumerable parts, themselves divided innumerable times" (De gen. et corr. 316a21-23). »

Sans parler des anachronismes et de l'exploitation contestable des sciences modernes (les mathématiques contemporaines, l'informatique, le traitement automatique des langues). L'auteur est manifestement plus à l'aise avec les mathématiques qu'avec l'histoire de la philosophie. Cependant, même dans ce domaine, il n'est pas à l'abri des erreurs. Il formalise la proposition 7.12 d'Euclide ainsi : « if $A: B=C: D$, then $(A+C)=(B+D)$ » (p. 156) ! Euclide ne dit pas une telle horreur, mais que si $\mathrm{A}: \mathrm{B}=\mathrm{C}: \mathrm{D}$, alors $\mathrm{A}: \mathrm{B}=(\mathrm{A}+\mathrm{C}):(\mathrm{B}+\mathrm{D}) \ldots$

Claire LOUGUET

Lisa Atwood Wilkinson, Parmenides and To Eon. Reconsidering Muthos and Logos, Continuum International Publishing Group, London-New York 2009, 176 p., ISBN 978-1-84706-245-1, € 108,60.

Tracciare una via verso Parmenide è quanto esplicitamente si propone la ricerca di Atwood Wilkinson: più esattamente un tentativo di «ascoltare di nuovo la sapienza dei nostri primi testi filosofici» (p. 7). Quella del poeta-filosofo di Elea, infatti, è opera della quale troppo spesso le letture metafisiche e analitiche dimenticano la peculiare collocazione tra la cultura orale della antica tradizione poetica e la allora relativamente recente pratica della scrittura: da quella tradizione, in effetti, il Peri physeōs, composto in esametri dattilici, assume non solo la forma del verso, ma anche il costume del proemio, inteso a stabilire contesto, personaggi e temi della narrazione (se non anche della argomentazione). D'altra parte, il poema è anche e soprattutto un testo filosofico, che pone alcune delle questioni di fondo del confronto teorico nel pensiero greco posteriore (e non solo) : un'opera filosofica nella misura in cui faticosamente si sottrae al tradizionale esercizio poetico, affermando il registro della scrittura, ovvero un ibrido in cui si possa sospettare che la scrittura costituisca freno inibitore all'autentica esperienza sapienziale? L'ipotesi intorno a cui lavora Atwood Wilkinson è, appunto, che pensatori come Senofane e Parmenide siano da considerare, per mentalità e pratica culturale, più vicini alla tradizione orale della poesia che non alla concettualità dei filosofi posteriori (Platone e Aristotele in particolare), verso cui si tende per lo più a proiettarli.

In questa prospettiva, allora, un percorso verso Parmenide non può prescindere da un più generale sforzo di comprensione della tradizione orale greco-arcaica (da « un percorso verso Omero », come recita appunto il titolo del capitolo 1), le cui possibilità di successo sono apparse in passato fortemente compromesse : qualcuno (Mircea Eliade), disperando, ha riconosciuto che tale tradizione è per noi completamente perduta. In alternativa è stata delineata una soluzione paradossale, ricercandone eco all'interno della poesia omerica : l'epica omerica costituisce ciò che noi possiamo conoscere della pratica orale greco- 
arcaica - una sorta di preistoria della scrittura, come osserva l'autrice (p. 11-12) ; allo stesso tempo, però, essa costituisce anche la nostra prima testimonianza letteraria (la nostra fonte primaria per la tradizione orale greca arcaica non è dunque orale ma scritta!). Consapevole della difficoltà di estrarre l'orale dallo scritto, la Atwood Wilkinson ritiene tuttavia eccessivo retroiettare nel mondo culturale di Omero la posteriore opposizione platonica tra oralità e scrittura : considerando l'epica omerica come una sorta di sistema comunicativo ibrido, sarebbe dunque almeno possibile tentare di identificare e separare gli elementi letterari della trascrizione dalla tradizione orale vera e propria.

Elaborando essenzialmente le tesi di classici della letteratura secondaria (Parry, Vernant, Havelock, Detienne, Nagy), Atwood Wilkinson valorizza due presupposti dell'esperienza che fa da sfondo alla prova poetica di Parmenide : il discorso cantato (sung speech, Detienne) - la cui funzione è di far accadere ciò che è reale, di modificare la realtà, non di descriverla - e la performance (Nagy), l'esecuzione poetica in cui l'interazione del bardo con la sua audience risulta fondamentale. Un aspetto, quest'ultimo, di recente sviluppato nella originale interpretazione del poema di Parmenide proposta da Chiara Robbiano (Becoming Being. On Parmenides' Transformative Philosophy, Academia Verlag, Sankt Augustin 2006), di cui, tuttavia, non si trova traccia nel lavoro della Atwood Wilkinson.

Come ella ci ricorda (p. 67), il discorso cantato è pratica comunicativa che, intrecciando linguaggio delle parole e linguaggio delle azioni, celebra la possibilità di una «comunicazione vera », di un «autentico contatto » (Vernant) con il divino. Suoni e gesti nel discorso cantato non rivestono funzioni segniche, non sono segnali di qualcosa ; essi, piuttosto, sono semata nel significato arcaico dell' espressione: presentano, realizzano ciò che non è immediatamente visibile, coincidono tendenzialmente (metaforicamente) con esso (p. 65-66). In questo senso il discorso cantato non si riferisce a un evento, ma è evento esso stesso.

Richiamare la peculiare matrice performativa pubblica del discorso cantato, significa inoltre evocare - accanto alla sua costruzione formulaica (Parry) - la centralità della memoria, il medium attraverso cui il divino ispira le parole del bardo, la musa trova espressione nel canto. Essa, fondamentalmente, secondo la lezione di Vernant, fa del poeta omerico un maestro di verità (Detienne), ne caratterizza l'eminenza rispetto ai molti (hoi pollor): una condizione che - come correttamente rileva l'autrice (p. 29) - ancora impronterebbe le figure dei poeti-filosofi Senofane e Parmenide (riconosciuti come hoi sophoi).

Dopo l'articolata analisi introduttiva (povera tuttavia di riscontri testuali diretti) dedicata alla forma comunicativa omerica (capitolo 2), alla sua ripresa in Senofane (capitolo 3), e alla discussione contemporanea (capitolo 4), negli ultimi due capitoli dell'opera Atwood Wilkinson si concentra sul poema di Parmenide. In conseguenza dello spazio destinato alla contestualizzazione, era plausibile attendersi un confronto stringente con il testo e le sue ambiguità; in realtà, anche in questa occasione, l'autrice privilegia la schermatura del dibattito interpretativo, pur arricchendolo con una serie di rilievi che è opportuno non trascurare.

Il primo riguarda la struttura conservata del Peri physeōs. Il Proemio ne costituisce il «commento preliminare» (p. 70), ciò che stabilisce quanto ha da venire : inteso spesso come cornice narrativa, se ne è per lo più misconosciuto il valore 
filosofico. Eppure allusioni all'epica e accenni religiosi sono reiterati fino a B19, quindi distribuiti per tutto quel che rimane del poema. Non è allora fuorviante leggere $\mathrm{i}$ tre segmenti (tradizionalmente identificati dalla Atwood Wilkinson come Proemio, Via della Verità e Via della Apparenza) in cui suddividiamo l'opera quasi fossero tra loro incommensurabili ? Perché i versi proemiali non dovrebbero essere degni di analisi? Secondo l'autrice, la svalutazione del Proemio scaturirebbe dalla distinzione post-platonica tra filosofia e poesia, in termini arcaici tra logos e mythos: ma guardando al testo senza preconcetti, dal momento che la dea introduce la propria comunicazione proprio all'interno del Proemio, ha senso assumere tale distinzione ? Giustamente Atwood Wilkinson rileva l'ambiguità della situazione interpretativa (p. 78) : da un lato si insiste spesso a demarcare l'argomentazione della Via della Verità dalla narrazione del Proemio; dall'altro, però, si continua ad associare le immagini del Proemio a quegli argomenti.

Proprio dal Proemio, dai suoi contorni indeterminati, muove un secondo rilievo su cui riflettere, in considerazione soprattutto della contestualizzazione culturale e della destinazione performativa del poema: la dea non nomina se stessa, né descrive se stessa o la casa in cui risiede, né indica l'argomento del suo discorso. Il suo è un mythos senza nomi. La dea sottolinea : « ti dirò e tu ascolta $\mathrm{e}$ riferisci ». Non c'è nulla da vedere per il giovane ; ci sono solo parole da ascoltare. Atwood Wilkinson invita allora a provare ad ascoltare il poema, ovvero a leggerlo in silenzio o a voce alta: l'effetto sarebbe quello di evidenziare la persistente presenza (aurale e visiva) di esti (senza soggetto), che, in particolare in B8, sembra « rompere la sintassi del passo » (p. 94), le regolari relazioni semantiche e le relazioni logiche o strutturali. Ascoltando il poema si diventerebbe dunque consapevoli dell'effetto ricercato da Parmenide : dissoluzione del ritmo (suono), che in un contesto orale si accompagna a dissociazione di significato ; entrambe scandite dalla costante ripetizione di esti ed einai.

Le deviazioni di Parmenide (in B8) dalle regole di composizione esametrica e dalle aspettative di ritmo e variazioni sonore (già segnalate da Mourelatos, come viene ricordato a p. 96) sarebbero indice, secondo l'autrice, dell'affacciarsi di un nuovo approccio di pensiero, che il linguaggio e i materiali della poesia orale non potevano sopportare. La prima lezione del poema potrebbe essere dunque: l'« è » (senza nome, immagine e soggetto) resiste a ogni tentativo di nominarlo. Scopo della ricerca (quest) di Parmenide sarebbe allora valorizzare l'abilità di pensare rispetto all'impulso di nominare. In tal senso, una quest il cui obiettivo sia raggiungere « è » non sarebbe nemmeno necessaria : il kouros non ha bisogno di viaggiare verso la Casa della dea, né di ascoltare il suo discorso ; egli già risiede in ciò-che-è e dunque pensa già ciò-che-è, comunque e sempre, anche senza intercessione della dea (p. 99).

Nel contesto Atwood Wilkinson propone un corollario interessante alla sua ipotesi : a dispetto dei modelli spesso evocati dalla critica, uno dei propositi del poema di Parmenide potrebbe essere proprio quello di dissolvere $i$ riferimenti epici letterali, mettendo in discussione l'idea stessa che una quest debba corrispondere a un viaggio o a una narrazione (p. 100). In questa prospettiva, possiamo forse concludere che forma poetica e contenuto drammatico si rivelino comunque 
funzionali a una intenzione educativa che il poema potrebbe aver mutuato dalla tradizione epica: plasmare l'atteggiamento critico dell'audience, modificandone consapevolezza e modo d'essere. Un risultato su cui, con diversa profondità, privilegiando l'analisi testuale e la contestualizzazione storico-filosofica, concordano i recenti studi della già citata Robbiano e della compianta Martina Stemich (Parmenides' Einübung in die Seinserkenntnis, Ontos Verlag, Frankfurt 2008).

La peculiare ottica adottata consente all'autrice di valorizzare anche alcuni aspetti trascurati della Via della Apparenza, quella parte del poema che, come ricordato, "l'audience di Parmenide ascoltava [...] per un periodo di tempo sostanzialmente più lungo » (p. 104), e in cui il vocabolario - come nel Proemio è ancora omerico, con resti di dizione formulaica.

Senza troppo intrattenersi sulle implicazioni e sulle alternative interpretative, Atwood Wilkinson si appella alla krisis di B2 e al suo esito (esclusione della via « non-è » come opzione reale) per sostenere una lettura « forte » della Doxa parmenidea : dal momento che le «opinioni mortali» non possono sorgere da una ontologia del "non-è », la dea non criticherebbe i mortali perché percepiscono scorrettamente, piuttosto perché denominano scorrettamente quello che percepiscono (p. 105). L'errore di fondo attribuito a quell'opinare in conclusione di B8 consisterebbe dunque nel contrapporre l'autorità dei nomi mortali alla costante autorità di « è ».

Suggestiva in questo senso l'ipotesi secondo cui l'espressione «l'ordinamento ingannevole delle mie parole » (kosmon emeōn epeōn apatēlon, B8.52) si riferirebbe appunto all'ordine verbale del discorso divino (e non ai contenuti delle opinioni introdotte), segnalerebbe il ritorno del linguaggio della dea alla semantica convenzionale e ordinaria e alle relazioni sintattiche caratteristiche del Proemio. Proprio il ripristino del ritmo poetico consueto doveva rendere ancora più esplicita all'esperienza di ascolto (dell'intero poema) l'assenza di nome per (the namelessness of) to eon. Parmenide allora sarebbe stato interessato a far emergere una duplice consapevolezza nel suo pubblico : (i) quella della costante presenza di «è̀ e (ii) quella dell'impulso a nominare, categorizzare, alla base delle credenze umane e della loro distorsione della realtà.

Difficilmente il contributo di Atwood Wilkinson potrà segnare una svolta nel campo degli studi sul Peri physeōs: troppo generale l'approccio, astratto dalla concreta problematicità del testo parmenideo, e probabilmente troppo monocorde, nell'insistenza sugli effetti della (unica) chiave « aurale». All'autrice dobbiamo, tuttavia, il richiamo fondamentale a una esperienza culturale per lo più trascurata o scarsamente valorizzata e la proposta di un punto di vista che scuote molte letture consolidate. Un peccato che nella ricerca non abbiano trovato spazio alcuni spunti emersi nel dibattito dell'ultimo decennio (ben documentato nelle raccolte Qu'est-ce que la philosophie présocratique?, a cura di A. Laks e C. Louguet, Presses Universitaires du Septentrion, Villeneuve d'Ascq 2002; Frühgriechisches Denken, a cura di G. Rechenhauer, Vandenhoeck \& Ruprecht, Göttingen 2005 ; La costruzione del discorso filosofico nell'età dei Presocratici, a cura di M.M. Sassi, Edizioni della Normale, Pisa 2006), segnatamente le indagini di M. Laura Gemelli Marciano sul contesto culturale presocratico e Patricia Curd 
sui «Presocratici come filosofi» (nella raccolta di Laks e Louguet). Avrebbero potuto suggerire una ricostruzione più equilibrata della fisionomia del poema.

Dario ZUCCHELLO

Lambros Couloubaritsis, La pensée de Parménide (en appendice traduction du Poème), Éditions Ousia, Bruxelles, 2008, 570 p. ISBN 978-2-87060-142-6

Nel caso di riedizione di opera ormai classica, normalmente le ragioni che hanno spinto l'autore a riproporne o rivederne l'impianto sono proposte nella nuova prefazione o in introduzione: non è facile, invece, imbattersi - anche all'interno del corpo del testo vero e proprio - nella esplicita confessione del travaglio teorico all'origine del ripensamento, della rielaborazione e della revisione delle proprie posizioni interpretative. È quello che accade affrontando la lettura della recente terza rivisitazione di Mythe et Philosophie chez Parménide di Lambros Couloubaritsis, così ampia da giustificare una titolazione più comprensiva e impegnativa: La pensée de Parménide.

Quasi alla conclusione del Capitolo II (Le Proème comme producteur de chemins), nel contesto di un confronto articolato e puntuale con le interpretazioni più recenti circa natura e funzione del primo segmento testuale del poema, l'autore, interrogandosi sul rischio che la lettura puramente metaforica delle figure di Dike, Ananke, Moira (e Aletheia) possa comportare la riduzione della nozione stessa di Eon (di cui quei numi sono posti a garanzia) a «mera finzione», osserva: "Cette question qui nous trouble depuis quelques années, et qui a suscité en nous le besoin de repenser la question du mythe, s'installe [...] comme une énigme, une aporie qu'on ne peut plus laisser dans l'ombre où elle se plaît à résider, inviolable comme l'Eon parménidien» (p. 191). Insomma, il nodo che Couloubaritsis ancora individua come essenziale per l'ermeneusi del poema è lo stesso all'origine della prima ricerca e di altri suoi importanti contributi parmenidei (Les multiples chemins de Parménide, in Études sur Parménide, sous la direction de P. Aubenque, Tome II: Problèmes d'interprétation, Vrin, Paris 1987, p. 25-43): il nesso tra gli echi del mito, a partire dal Proemio, e la struttura teorica dell'opera. L'attenzione a questo tema si è poi tradotta nello sviluppo di due fondamentali (e complementari) direttrici d'indagine: la "deontologizzazione" della interpretazione del poema e il recupero della centralità della sezione sulla Doxa come tentativo di fondazione di una nuova «fisica».

Rispetto alle edizioni precedenti colpiscono positivamente due elementi di fondo della ricerca: l'ampiezza e la profondità della prospettiva, in altre parole la pluralità di punti di vista e lo spettro culturale considerati, insieme allo spessore della dimensione diacronica, che tende a collocare il pensiero di Parmenide saldamente entro la tradizione del pensiero e della letteratura arcaica, proiettandolo verso Platone e Aristotele. Risalta allora significativamente l'assenza totale, nell'aggiornamento del dibattito e nella costruzione della linea di lettura, del riferimento ai contributi di Peter Kingsley - Ancient Philosophy, Mystery and Magic. Empedocles and the Pythagorean Tradition (1995), In the Dark Places of Wisdom (1999), Reality (2003) - che negli ultimi due decenni hanno tracciato una interessante alternativa, radicando e retroiettando piuttosto l'Eleate all'interno di un passato 